\title{
Detection Capability Verification and Performance Test for the High Resolution Imaging Camera of China's Tianwen-1 Mission
}

\author{
Wei Yan ${ }^{1}$ (D) Jianjun Liü ${ }^{1,2} \cdot$ Xin Ren $^{1} \cdot$ Chunlai $\mathrm{Li}^{1,2}$. Qiang Fu ${ }^{1}$ Dong Wang ${ }^{3}$. \\ Jihong Dong ${ }^{3} \cdot$ Xiaoxia Zhang $^{1} \cdot$ Wangli Chen $^{1} \cdot$ Xu Tan $^{1} \cdot$ Hai Huang $^{1} \cdot$ Zhirui Cao $^{3}$. \\ Dejie $\mathrm{Yan}^{3} \cdot \mathrm{Qi} \mathrm{Ji}{ }^{3} \cdot$ Fanlu $\mathrm{Wu}^{3}$
}

Received: 29 September 2020 / Accepted: 26 July 2021 / Published online: 16 August 2021

(c) The Author(s) 2021

\begin{abstract}
High-resolution optical cameras have always been important scientific payloads in Mars exploration missions, which can obtain detailed images of Martian surface for the study of geomorphology, topography and geological structure. At present, there are still many challenges for Mars high-resolution images in terms of global coverage, stereo coverage (especially for colour images), and data processing methods. High Resolution Imaging Camera (HiRIC) is a high-quality, multi-mode, multi-functional, multi-spectral remote sensing camera that is suitable for the deep space developed for China's first Mars Exploration Mission (Tianwen-1), which was successfully launched in July 2020. Here we design special experiments based on the in-orbit detection conditions of Tianwen-1 mission to comprehensively verify the detection capability and the performance of HiRIC, from the aspects of image motion compensation effect, focusing effect, image compression quality, and data preprocessing accuracy. The results showed that the performance status of HiRIC meets the requirements of obtaining high resolution images on the Martian surface. Furthermore, proposals for HiRIC in-orbit imaging strategy and data processing are discussed to ensure the acquisition of high-quality HiRIC images, which is expected to serve as a powerful complementation to the current Mars high-resolution images.
\end{abstract}

Keywords Tianwen- $1 \cdot$ High resolution imaging camera $\cdot$ Ground scientific verification test · Detection capability · Imaging strategy

The Huoxing-1 (HX-1) / Tianwen-1 (TW-1) mission to Mars

Edited by Chunlai Li and Jianjun Liu

\footnotetext{
$\triangle$ W. Yan

yanw@nao.cas.cn

1 Key Laboratory of Lunar and Deep Space Exploration, National Astronomical Observatories, Chinese Academy of Sciences, Beijing 100101, China

2 University of Chinese Academy of Sciences, Beijing 100049, China

3 Changchun Institute of Optics, Fine Mechanics and Physics, Chinese Academy of Sciences, Changchun 130033, China
} 


\section{Introduction}

Image and topography data are some of the most important exploration data of planetary and deep space exploration, and are also the important basic for studying the internal structure and surface evolution history of terrestrial planets (Carr 2007; Ren et al. 2019). Due to the lack of prior knowledge and experience in the complexity of the Mars exploration environment, high-resolution optical cameras have always been important scientific payloads in international Mars exploration missions. As the first spacecraft to orbit another planet, Mariner 9 contributed greatly to the exploration of Mars. After the occurrence of dust storms on the planet for several months following its arrival, Mariner 9 successfully returned 7329 images over the course of its mission, which concluded in October 1972. These images covered $85 \%$ of Martian surface at resolutions from 1 kilometer per pixel to 100 meters per pixel (NASA 2011, 2019). The findings from the Mariner 9 mission underpinned the later Viking program. Then Viking $1 \& 2$ returned images of the Martian surface with a spatial resolution of about $300 \mathrm{~m}$. The first truly high-resolution images of Martian surface came from the Mars Orbiter Camera (MOC) on Mars Global Surveyor (MGS). MOC returned narrow angle images covered $5.45 \%$ of the Martian surface at better than $12 \mathrm{~m} / \mathrm{pixel}$, and $\sim 0.5 \%$ of the Mars at better than $3 \mathrm{~m}$ /pixel by the mission's end (Malin et al. 1992, 2010). The High Resolution Imaging Science Experiment (HiRISE, McEwen et al. 2007, 2010) and the Context Camera (CTX, Malin et al. 2007) are two high resolution cameras on Mars Reconnaissance Orbiter (MRO) launched in 2005. HiRISE has 14 charge-coupled devices (CCDs), each with two output channels, and with multiple choices for pixel binning and number of Time Delay and Integration (TDI) lines, to provide detailed images of the Martian surface with an original spatial resolution of 0.25 to $1.3 \mathrm{~m} / \mathrm{pixel}$, in which $0.3 \mathrm{~m}$ is currently the highest spatial resolution of the Martian surface images (Lefort et al. 2009; Dundas et al. 2010; Bondarenko et al. 2014). CTX is intended to provide context images for data acquired by other MRO instruments, covering 9\% of the Mars at $6 \mathrm{~m} /$ pixel, but the number of overlapping images (for stereo coverage, image mosaics, etc.) will reduce this percentage (Malin et al. 2007; Thomas et al. 2017). The High Resolution Stereo Camera (HRSC, Neukum and Jaumann 2004; Jaumann 2007) on the Mars Express mission and the Colour and Stereo Surface Imaging System (CaSSIS, Thomas et al. 2017) on ExoMars Trace Gas Orbiter (TGO) are high resolution cameras for European Space Agency (ESA)' s Mars exploration mission. HRSC is unique in terms of its imaging principle, in that concomitant digital terrain models (DTMs) and multiple orthoimages can be derived based on its standard mode of operation, and in its specific geometric resolution. HRSC images obtained by its 9 color CCD line sensors mounted in parallel have almost achieved Mars global coverage, in which the spatial resolution of images covering $70 \%$ of the Martian surface reaches $10 \sim 20 \mathrm{~m}$, and the spatial resolution of images in $97 \%$ of the coverage area is better than $100 \mathrm{~m}$ (Gwinner et al. 2016). Moreover, an additional Super Resolution Channel (SRC) of HRSC can provide co-aligned images at approximately 5 times higher resolution (Oberst et al. 2008). CaSSIS is intended to acquire moderately high resolution ( $4.6 \mathrm{~m} / \mathrm{pixel})$ targeted images of the Mars at a rate of 10 20 images per day from a roughly circular orbit $400 \mathrm{~km}$ above the surface, which can be comparable or superior to all currently operational orbiting cameras apart from HiRISE, and only MOC had superior resolution (Thomas et al. 2017).

Up to this time, high-resolution cameras for international Mars exploration missions have acquired significant numbers of images on the Martian surface, which have greatly enriched our understanding of the Mars (Keszthelyi et al. 2008; Oberst et al. 2008; Malin et al. 2010; McEwen et al. 2010; Thomas et al. 2017). However, the current high-resolution images of the Martian surface still have many challenges worthy of attention. For example, 

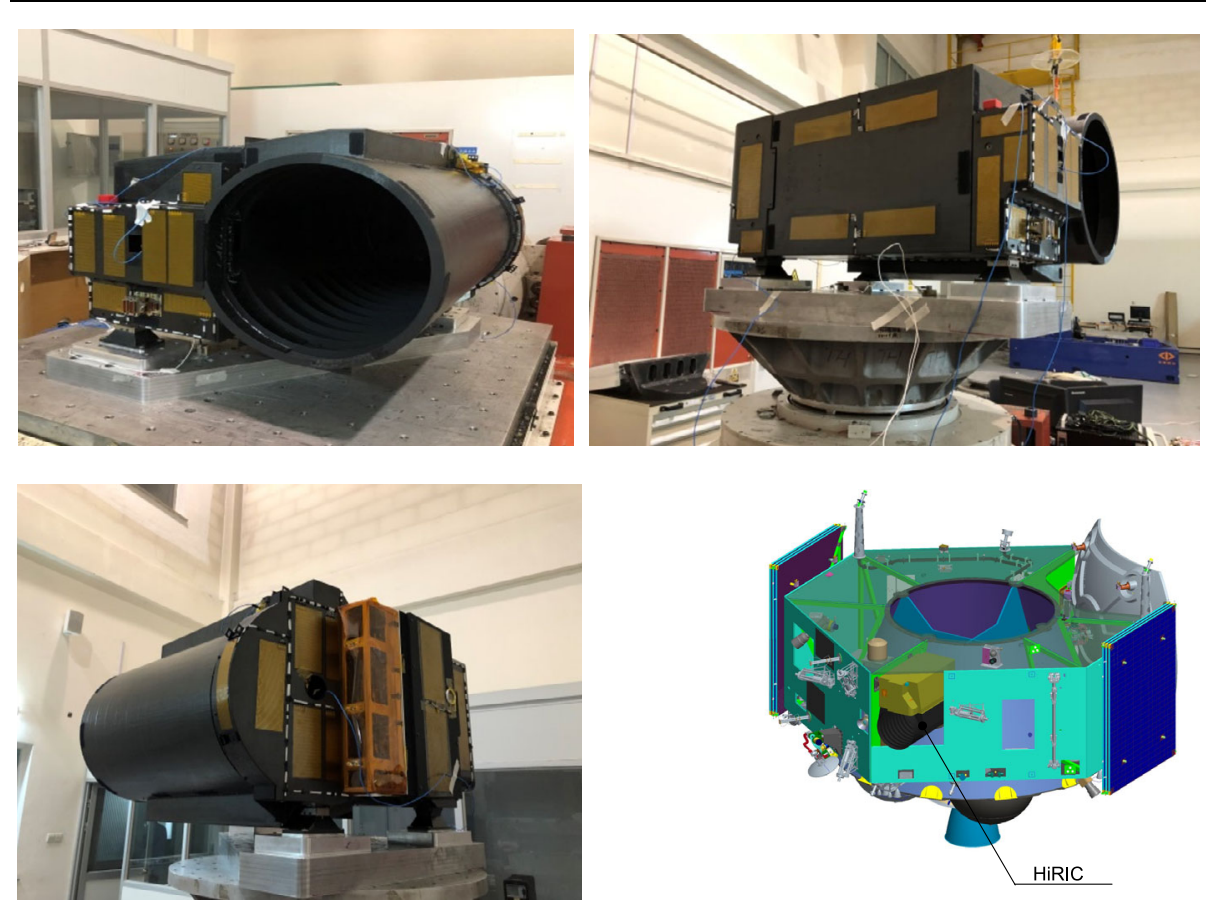

Fig. 1 HiRIC and its installation position on Tianwen-1 Orbiter

the coverage (especially stereo coverage) of high-resolution images at meter and sub-meter level is very limited. Simultaneously, HiRISE has absolute radiometric calibration challenges because of highly variable focal-plane temperatures (Delamere et al. 2010). HRSC has provided $>90 \%$ global stereo and colour coverage, but the highly elliptical orbit and wide-angle optics result in $>10 \mathrm{~m} /$ pixel scale at best. Also, HRSC images are acquired at different emission and phase angles per colour making generation of photometrically accurate colour products at best difficult (McCord et al. 2007). CaSSIS, which has a higher resolution, has just started its work for a short time and its final Mars coverage effect needs further evaluation. Therefore, in terms of data complementation, there are still a lot of work to do for the subsequent Mars exploration mission.

China's first Mars exploration mission (Tianwen-1) has been successfully launched an orbiter and a rover (named as Zhurong) in July 2020 to conduct a global and comprehensive exploration of the Mars, and to carry out regional patrolling on the Martian surface (Ye et al. 2017). Mars morphology and geological structure is also one of its the main scientific objectives. In order to achieve this objective, a High Resolution Imaging Camera (HiRIC, Fig. 1) is mounted on Tianwen-1 Orbiter to obtain detailed optical images of interest areas on the Martian surface to study the surface topography and changes of the Mars, the identification of characteristic geomorphic units, as well as geological structure, such as landform of water, volcano, impact crater, erosion, etc. A comprehensive verification of the detection capability and performance of HiRIC on the ground facilitates the acquisition of high-quality images during its in-orbit operation.

In this paper, an overview of HiRIC is introduced in Sect. 2; Sect. 3 designed special ground scientific verification experiments based on the in-orbit detection conditions of Tianwen-1 mission to comprehensively verify the detection capability and the performance 
of HiRIC; Sect. 4 analyzes the image quality according to the test results; Sect. 5 evaluates the actual detection capability of HiRIC during in-orbit operation. Finally, some proposals for in-orbit imaging strategy are given.

\section{The Instrument}

The HiRIC is one major payload of China's Tianwen- 1 mission. It adopts an Off-Axis ThreeMirror Astigmatic (TMA) optical system with a focal length of $4640 \mathrm{~mm}$, a F-number of 12 and a Field of View (FOV) of $2^{\circ} \times 0.693^{\circ}$. In order to reduce the instrument weight, carbonbased material is widely used in the opto-mechanical structure which is in ultra-lightweight design (seen in Table 1, Meng et al. 2021).

HiRIC contains three linear- array TDI CCDs and two area- array Complementary Metal Oxide Semiconductors (CMOS) on one imaging focal plane to produce push-scan and frame imaging respectively (seen in Fig. 2). TDI CCDs can work in panchromatic and 4 spectral bands, including blue (B1), green (B2), red (B3), and near-infrared (B4), so that HiRIC can obtain both panchromatic and color images.

The pixel number for the panchromatic band of each TDI CCD in the cross-scan direction is 6144 with a pixel size of $8.75 \mu \mathrm{m} \times 8.75 \mu \mathrm{m}$, while the pixel number for the color bands is 1536 with a pixel size of $35 \mu \mathrm{m} \times 35 \mu \mathrm{m}$. The maximum TDI integration levels for panchromatic and color bands are 96 and 64 respectively. Each two staggered CCDs are mechanically spliced with an overlap of about 116 pixels (design value) for panchromatic band so that the equivalent pixel number of the spliced TDI CCD is 18200, corresponding to a $9 \mathrm{~km}$ swath width at the altitude of $265 \mathrm{~km}$. CMOS detectors are working in an electronic rolling imaging mode. The effective pixel number for each CMOS detector is $512 \times 512$ with a pixel size of $44 \mu \mathrm{m} \times 44 \mu \mathrm{m}$. Detailed radiometric and geometric calibration of the detectors has been carried out during the development of HiRIC (Meng et al. 2021).

The HiRIC is mainly used to obtain detailed optical images of interest areas on the Martian surface when the orbital altitude is changed between $265 \mathrm{~km}$ and $800 \mathrm{~km}$ and the solar elevation angle is greater than $10^{\circ}$ (our goal is to image when the solar elevation angle is greater than $5^{\circ}$ ). There are 4 working modes for HiRIC, including imaging, health checking, thermal controlling, and focusing, in which imaging (as shown in Table 2) is its main scientific exploration mode during in-orbit operation. It can be seen that HiRIC's imaging mode has very flexible combination settings to meet different detection requirements. Because of the highest ground spatial resolution of $0.5 \mathrm{~m}$ on the Martian surface and the image swath width of $9 \mathrm{~km}$, panchromatic band CCD push-scan imaging will be the default in-orbit detection mode for HiRIC, while other imaging mode like CMOS will hardly be used. Therefore, this paper will focus on the performance and detection capability of CCD detectors.

China's first Mars mission can be divided into launch phase, Earth-Mars transfer phase, Mars capture orbit phase, Mars parking orbit phase, EDL (Entry, Descent, Landing) phase and scientific exploration phase (Li et al. 2021), in which scientific exploration phase includes relay and survey orbit and global reconnaissance orbit. HiRIC will begin its work in the Mars parking orbit with orbital altitudes of about $280 \mathrm{~km}$ and $59000 \mathrm{~km}$ at the periareon and apoareon, respectively, and an orbital period of about 2 days, when it will obtain stereo images of the primary candidate landing area (Meng et al. 2021). Global reconnaissance orbit phase is another working period for HiRIC, which is also a large elliptical orbit with orbital altitudes of about $265 \mathrm{~km}$ and $11932 \mathrm{~km}$ at the periareon and apoareon, respectively, an orbital inclination of $90^{\circ} \pm 5^{\circ}$, and an orbital period of 7.8 hours. Global reconnaissance 
Table 1 Specifications of HiRIC

\begin{tabular}{|c|c|c|c|}
\hline No. & Parameter & & Specifications \\
\hline 1 & $\begin{array}{l}\text { Ground resolution }(\mathrm{m} / \mathrm{pixel} \text {, } \\
\text { at the altitude of } 265 \mathrm{~km})\end{array}$ & $\begin{array}{l}\text { panchromatic } \\
\text { colors }\end{array}$ & $\begin{array}{l}>2.5 \mathrm{~m} \text {, interest areas are better than } 0.5 \mathrm{~m} \\
>10 \mathrm{~m} \text {, interest areas are better than } 2.0 \mathrm{~m}\end{array}$ \\
\hline 2 & Field of View (FOV) & & $2^{\circ} \times 0.693^{\circ}$ \\
\hline 3 & F-number & & 12 \\
\hline 4 & Focal length (mm) & & 4640 \\
\hline 5 & $\begin{array}{l}\text { Swath width }(\mathrm{km} \text {, at the } \\
\text { altitude of } 265 \mathrm{~km})\end{array}$ & & 9 \\
\hline 6 & MTF(at Nyquist frequency) & & $>0.18$ \\
\hline 7 & $\begin{array}{l}\text { Signal- to-noise ratio (SNR, } \\
\text { in the case of reflectance of } 0.2 \\
\text { and solar elevation angle } \\
\text { of } 30^{\circ} \text { ) }\end{array}$ & & $>100$ \\
\hline 8 & Spectral wavelength $(\mu \mathrm{m})$ & panchromatic & $0.45 \sim 0.9$ \\
\hline 9 & & colors & $\begin{array}{l}\text { B1:0.45 0.52 } \\
\text { B2:0.52 0.60 } \\
\text { B3:0.63 0.69 } \\
\text { B4:0.76 0.90 }\end{array}$ \\
\hline 10 & Bits per pixel & & $12 \mathrm{bit}$ \\
\hline 11 & In-orbit image processing & & $\begin{array}{l}\text { Image resampling (including binning and sampling); } \\
\text { Image windowing; } \\
\text { Band selection }\end{array}$ \\
\hline 12 & Compression & & $\begin{array}{l}\text { Non-compression, lossless compression, compression } \\
\text { at } 3: 1 / 4: 1 / 6: 1 \text { (default) /12:1/15:1 }\end{array}$ \\
\hline 13 & Weight $(\mathrm{kg})$ & & 42 \\
\hline
\end{tabular}

orbit phase will last about 15 months after the rover Zhurong has conducted 90 sols of in-situ exploration on the Martian surface. As the probe orbital altitude varies between $265 \mathrm{~km}$ and $800 \mathrm{~km}$, the operating distance range of HiRIC, the subsatellite velocity of the Tianwen-1 Orbiter will vary between approximately $4 \mathrm{~km} / \mathrm{s}$ and $3 \mathrm{~km} / \mathrm{s}$. The variation of imaging distance and subsatellite velocity will affect the imaging quality of HiRIC, so the image motion compensation and focusing are very important for the HiRIC image effect.

On the other hand, since the fastest subsatellite velocity of the Tianwen-1 Orbiter is about $4 \mathrm{~km} / \mathrm{s}$ at the altitude of $265 \mathrm{~km}$ (at the periareon), the data acquisition rate of HiRIC will reach the maximum of $1695.94 \mathrm{Mbps}$ under the default imaging mode. HiRIC is planned to work about $30 \mathrm{~s} \sim 45 \mathrm{~s}$ per time so the maximum data volume will be $74.53 \mathrm{~Gb}$. Over the course of one Martian year orbital exploration, the distance from Tianwen-1 Orbiter to the Earth will vary between 81 million and 394 million $\mathrm{km}$, with designed data transmission rate to ground fluctuating between $2048 \mathrm{kbps}$ and $128 \mathrm{kbps}$. Considering the visual conditions between the ground station and the Orbiter, the maximum data transmission time is just about $5 \sim 6$ hours per day. The data transmission capacity of Mars exploration is limited. Thanks to the 70-meter diameter antenna newly built in Wuqing by the Ground Research and Application System (GRAS, Wu et al. 2013; Liu et al. 2018) of Tianwen-1 mission, the actual data transmission rate is expected to nearly double the design number. Even so, the daily data transmission time is still difficult to meet the downlink demand of HiRIC raw detection data. Therefore, data compression of is necessary HiRIC images. 


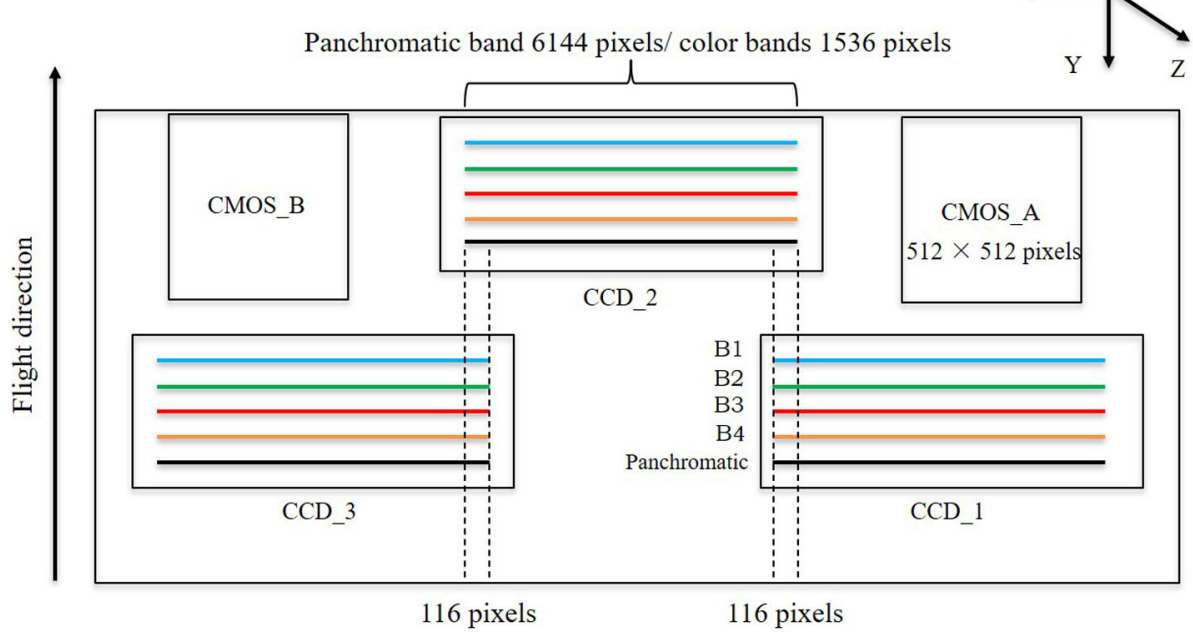

Fig. 2 Distribution of HiRIC CCDs and CMOS detectors on the focal plane. The probe flight direction is from the FOV of $-0.1^{\circ}$ to $+0.535^{\circ}$, and the TDI CCD integral direction is from the blue imaging area (B1) to the panchromatic imaging area. Coordinate system XYZ is the ground calibration coordinate system of HiRIC, in which the $\mathrm{X}$-axis is parallel to the line array direction of CCDs, the Z-axis is perpendicular to the focal plane, and the $\mathrm{Y}$-axis completes a right-handed system with the $\mathrm{X}$-axis and $\mathrm{Z}$-axis

\section{Image Quality Influencing Factors and Verification Experiments}

As previously stated, HiRIC will normally use the panchromatic TDI CCD band for pushscan imaging during in-orbit operation, just like HiRISE, HRSC and other similar international Mars exploration cameras. There are many factors that affect the image quality of HiRIC, including image motion and defocusing related to the large elliptical orbit of Tianwen-1 Orbiter together with the orbit determination accuracy and platform attitude accuracy, the ground albedo of the Martian surface and solar elevation angle related to the imaging targets, image compression ratio related to the data transmission capability, as well as the radiometric calibration coefficients accuracy and the CCD stitching accuracy related to data processing, etc.

In order to guarantee the performance of HiRIC, the development department, Changchun Institute of Optics, Fine Mechanics and Physics, Chinese Academy of Sciences (CIOMP), carried out detailed design and analysis of its optical system, mechanical structure, detector, thermal control, in-orbit data processing, etc., and carried out a number of calibrations including geometric calibration, radiometric calibration, spectral calibration, color calibration, etc., in the laboratory before the mission (Meng et al. 2021). In this paper, we will conduct ground-based scientific validation tests using HiRIC product to comprehensively evaluate and validate its performance and detection capabilities in the context of the orbital characteristics of the mission and the actual in-orbit operating conditions of HiRIC, as well as to assess the correctness and validity of the calibration results in the laboratory. The results also can be used to guide the in-orbit detection strategy and data processing.

The ground scientific verification test was organized and implemented by the GRAS. The development department of HiRIC and other related institutions are participated. The qualification model and flight model of HiRIC, which has been calibrated by radiation calibration and geometric calibration was used in this test. Two types of experiments are formulated, 
Table 2 Imaging modes of HiRIC

\begin{tabular}{|c|c|c|c|}
\hline No. & Modes & Definition & Working conditions \\
\hline \multirow[t]{2}{*}{2} & \multirow[t]{2}{*}{$\begin{array}{l}\text { Panchromatic } \\
\text { (Default) }\end{array}$} & $\begin{array}{l}\text { Pixel resolution: } \\
0.5 \mathrm{~m} @ 265 \mathrm{~km}\end{array}$ & $\begin{array}{l}\text { 1) } 3 \text { CCD images; } \\
\text { 2) Non-binning; } \\
\text { 3) Image resolution: } \\
0.5 \text { m@ } 265 \mathrm{~km} \text {; }\end{array}$ \\
\hline & & $\begin{array}{l}\text { Swath width: } \\
9.0 \mathrm{~km} @ 265 \mathrm{~km}\end{array}$ & $\begin{array}{l}\text { 4) Image Swath width: } 9 \text { km@ } 265 \mathrm{~km} \text {; } \\
\text { 5) Effective number of bits (ENOB): } 12 \text { bits }\end{array}$ \\
\hline \multirow[t]{2}{*}{2} & \multirow[t]{2}{*}{$\begin{array}{l}\text { Colors } \\
(\mathrm{B} 1 / \mathrm{B} 2 / \mathrm{B} 3)\end{array}$} & $\begin{array}{l}\text { Pixel resolution: } \\
2.0 \mathrm{~m} @ 265 \mathrm{~km}\end{array}$ & $\begin{array}{l}\text { 1) } 3 \text { CCD images; } \\
\text { 2) Non-binning; } \\
\text { 3) Image resolution: } 2.0 \text { m@ } 265 \text { km; }\end{array}$ \\
\hline & & $\begin{array}{l}\text { Swath width: } \\
9.0 \mathrm{~km} @ 265 \mathrm{~km}\end{array}$ & $\begin{array}{l}\text { 4) Image swath width: } 9 \text { km@ } 265 \mathrm{~km} \text {; } \\
\text { 5) ENOB: } 12 \text { bits }\end{array}$ \\
\hline \multirow[t]{2}{*}{3} & \multirow[t]{2}{*}{$\begin{array}{l}\text { Full spectrum } \\
\text { (Panchromatic, } \\
\text { B1/B2/B3/B4) }\end{array}$} & $\begin{array}{l}\text { Pixel resolution: } \\
\text { Panchromatic } \\
0.5 \mathrm{~m} / @ 265 \mathrm{~km} \\
\mathrm{BL} / \mathrm{RE} / \mathrm{GR} / \mathrm{IR} \\
2.0 \mathrm{~m} @ 265 \mathrm{~km}\end{array}$ & $\begin{array}{l}\text { 1) } 3 \text { CCD images; } \\
\text { 2) Non-binning; } \\
\text { 3) Image resolution: Panchromatic: } 0.5 \text { m@ } 265 \text { km; } \\
\text { B1/B2/B3/B4: } 2.0 \text { m@ } 265 \text { km }\end{array}$ \\
\hline & & $\begin{array}{l}\text { Swath width: } \\
9.0 \mathrm{~km} @ 265 \mathrm{~km}\end{array}$ & $\begin{array}{l}\text { 4) Image swath width: } 9 \mathrm{k} \text { m@ } 265 \mathrm{~km} \text {; } \\
\text { 5) ENOB:12 bits }\end{array}$ \\
\hline \multirow[t]{2}{*}{4} & \multirow[t]{2}{*}{ CMOS } & $\begin{array}{l}\text { Pixel resolution: } \\
2.5 \mathrm{~m} @ 265 \mathrm{~km}\end{array}$ & $\begin{array}{l}\text { 1) } 4 \times 4 \text { binning; } \\
\text { 2) Effective pixels: } 512 \times 512 \text {; }\end{array}$ \\
\hline & & $\begin{array}{l}\text { Swath width: } \\
1.2 \mathrm{~km} \times 1.2 \mathrm{~km} @ 265 \mathrm{~km}\end{array}$ & $\begin{array}{l}\text { 3) Image swath width: } 1.2 \mathrm{~km} \times 1.2 \mathrm{~km} @ 265 \mathrm{~km} \text {; } \\
\text { 4) ENOB: } 12 \text { bits }\end{array}$ \\
\hline 5 & Customize & Can be set & Can be set \\
\hline
\end{tabular}

including the laboratory simulation imaging experiments used for quantitative verification of image motion compensation (marked as A-1) and focusing effect (A-2), and the external field push-scan imaging experiments (B-1) used for simulating the imaging process of HiRIC and evaluating its detection capability and image quality. The details are as follows in Table 3.

\subsection{Laboratory Imaging Experiments}

\subsubsection{Image Motion Compensation Effect Evaluation Test}

Image motion is a common phenomenon in linear array push-scan cameras. It will cause different degrees of degradation of the dynamic Modulation Transfer Function (MTF) of images during in-orbit operation due to the orbit determination accuracy, platform attitude accuracy, and fluctuations of the Martian surface, etc. Image motion is difficult to eliminate, but its magnitude can be controlled within a reasonable range by compensation. The image motion of HiRISE images is usually less than half of a pixel (Lee et al. 2001; McEwen et al. 2010) and for HRSC, an exposure time of $0.6 \mathrm{~ms}$ will be required to avoid the image motion.

The image motion of HiRIC can be divided into forward image motion (along the flight direction) and lateral image motion (perpendicular to the flight direction) (Wang 1998). During the in-orbit operation, the image motion compensation parameters, including imaging frequency and the drift angle, will be calculated based on the predicted orbital parameters of the Orbiter and the Martian surface topography, to compensate for the image motion mentioned above. The higher the TDI integration levels, the more stringent the requirements 
Table 3 Scientific verification test for HiRIC

\begin{tabular}{|c|c|c|}
\hline No. & Contents & Explanation \\
\hline \multirow[t]{3}{*}{ A-1 } & $\begin{array}{l}\text { Image motion compensation } \\
\text { effect evaluation }\end{array}$ & $\begin{array}{l}\text { Carry out laboratory imaging experiments to obtain images } \\
\text { under ideal conditions where the image motion speed is } \\
\text { perfectly matched; }\end{array}$ \\
\hline & & $\begin{array}{l}\text { Analyze the effects of Orbiter altitude errors on image motion } \\
\text { compensation effects; }\end{array}$ \\
\hline & & $\begin{array}{l}\text { Evaluate the corresponding maximum error amount level } \\
\text { under the current in-orbit image motion compensation } \\
\text { scheme. }\end{array}$ \\
\hline \multirow[t]{3}{*}{ A-2 } & Focusing effect evaluation & $\begin{array}{l}\text { Carry out laboratory imaging experiments to obtain clear } \\
\text { images of the target at the optimal focal plane position of the } \\
\text { camera; }\end{array}$ \\
\hline & & Acquire images in the defocused state; \\
\hline & & $\begin{array}{l}\text { Perform focusing and obtain images again to compare image } \\
\text { quality before and after focusing. }\end{array}$ \\
\hline B-1 & $\begin{array}{l}\text { External field push-scan } \\
\text { imaging experiment }\end{array}$ & $\begin{array}{l}\text { Conduct an external field push-scan imaging test to verify the } \\
\text { entire in-orbit detection process and data processing of } \\
\text { HiRIC. Experimental images will be used to evaluate the } \\
\text { camera's compression quality, radiation correction effect, and } \\
\text { CCD splicing effect. }\end{array}$ \\
\hline
\end{tabular}

for the orbit determination accuracy. Because Tianwen-1 has a large elliptical orbit $(265 \times$ $11932 \mathrm{~km}$ for mission orbit) and its orbit determination accuracy is just several $\mathrm{km} \mathrm{s}$, the image motion compensation of HiRIC images will be severely influenced by the variable orbital altitude and orbit altitude measurement errors.

In the image motion compensation effect evaluation test, a spatial frequency grating with a spatial frequency of $57 \mathrm{lp} / \mathrm{mm}$, the same as the Nyquist frequency of HiRIC (Meng et al. 2021 ), was driven by a rotating motor with an angular velocity of $80 \% \sim 500 \%$ s to simulate a dynamic imaging target in the orbital altitude range of $265 \mathrm{~km} \sim 340 \mathrm{~km}$ relative to the HiRIC focal plane. HiRIC was placed in a vacuum tank and connected to this device via a collimator. The CCDs are aligned with the spatial frequency grating by an attitude adjustment platform and the target is imaged at an imaging frequency that matched the angular velocity of the rotating motor through the imaging control system (Seen in Fig. 3).

In order to comprehensively verify the image motion compensation effect of HiRIC, different working conditions are designed, including imaging frequency calculated by different methods (theoretical calculation and real-time calculation from orbital parameters), orbital parameters with different orbital altitude errors $(1 \mathrm{~km}, 2 \mathrm{~km}$ and $3 \mathrm{~km})$, different simulated orbital altitudes ( $265 \mathrm{~km}$ and $340 \mathrm{~km}$ ), different TDI integration levels (96 8), and so on. A total of 48 sequence images have been obtained to evaluate the image motion compensation effect of HiRIC. It should be noted that factors such as the production accuracy of the spatial frequency grating, assembly accuracy between HiRIC, rotating motor and collimator, will introduce errors to the test in addition to the design conditions.

\subsubsection{Focusing Effect Evaluation Test}

Unlike MRO and TGO, Tianwen-1 is designed as a large elliptical orbit with orbital altitudes of $265 \mathrm{~km}$ and $11932 \mathrm{~km}$ at the periareon and apoareon, respectively. To achieve clear imaging at different orbital altitudes, HiRIC has designed a focusing mirror (FM) for inorbit focusing. A focusing mechanism is designed to control the FM to achieve this function 


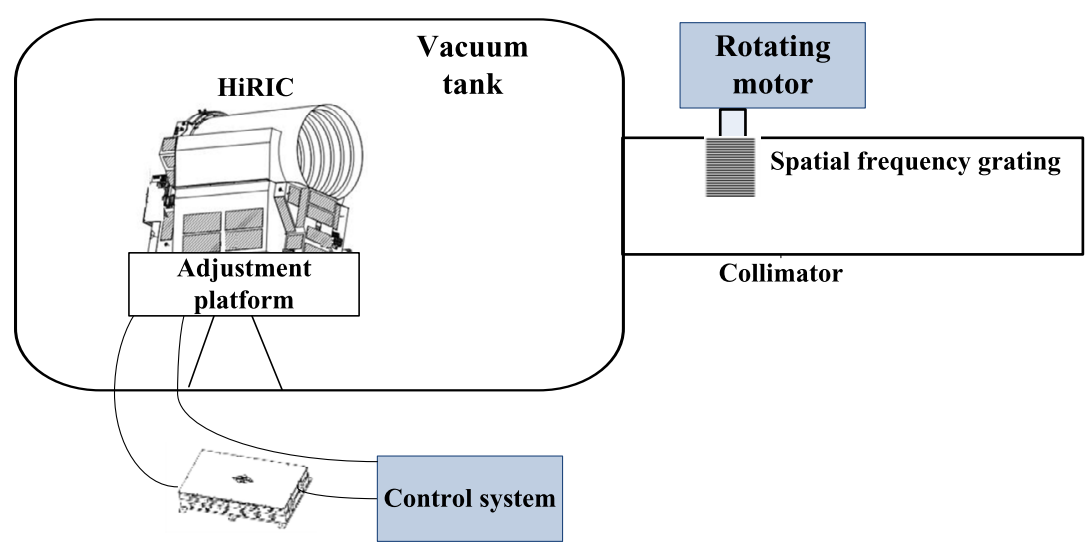

Fig. 3 Schematic diagram of image motion compensation effect evaluation test

(Meng et al. 2021), with a focusing stroke of $4 \mathrm{~mm}$ and a total focusing error of $4.7 \mu \mathrm{m}$. In fact, HiRIC's focal plane has already been positioned on the ground according to a distance target of $500 \mathrm{~km}$ to accommodate the imaging quality requirement when the orbital altitude varies between $265 \mathrm{~km}$ and $800 \mathrm{~km}$, i.e., to meet the working distance range of HiRIC. In-orbit focusing is only required in rare cases such as extended missions.

5 sequence images of a spatial frequency grating with a spatial frequency of $57 \mathrm{lp} / \mathrm{mm}$, which is the same as the Nyquist frequency of HiRIC, have been obtained in the focusing effect evaluation test through a collimator. These images include clear images of the spatial frequency grating at a simulated orbital altitude of $500 \mathrm{~km}$, corresponding defocused images at a simulated orbital altitude of $265 \mathrm{~km}$ and at infinity distance, and finally, images of the same target after focusing.

\subsection{External Field Push-Scan Imaging Experiment}

The purpose of external field push-scan imaging experiment is to verify the entire in-orbit detection process and data processing of HiRIC.

During the external field push-scan imaging experiment, HiRIC was placed horizontally on a turntable, which will be rotated clockwise (as viewed from the top of the turntable) according to the designed rotation angular velocity to simulate the in-orbit imaging process. The CCD Imaging commands for different operating conditions are generated according to the upload data format and sent to the camera through ground control system during the external field imaging test. Then the turntable will be rotated and HiRIC will image the far distance targets under the imaging commands. Images will be stored and transferred to the computer. Because the focal plane position of HiRIC has been set to be clearly visible to targets at a distance of $500 \mathrm{~km}$, in order to obtain clear images of the remote target within a few kilometers, the focal plane of the HiRIC qualification model was adjusted backward by $6.1 \mathrm{~mm}$ after theoretical calculations in this test. A total of 68 sequence images, an amount of data of $393 \mathrm{~Gb}$, have been obtained in this test. Images of this experiment can be used as reference for analyzing the quality of in-orbit images. 
Table 4 Dynamic MTF of HiRIC images in the image motion compensation effect test

\begin{tabular}{|c|c|c|c|c|c|c|}
\hline \multirow{3}{*}{$\begin{array}{l}\text { Orbital } \\
\text { altitude }(\mathrm{km})\end{array}$} & \multirow{3}{*}{$\begin{array}{l}\text { Integration } \\
\text { levels }\end{array}$} & \multicolumn{4}{|c|}{ Qualification model } & \multirow{3}{*}{$\begin{array}{l}\text { Flight model } \\
\text { Condition } 5 \\
\text { MTF/MTF } \\
\text { decline ratio }\end{array}$} \\
\hline & & Condition 1 & Condition 2 & Condition 3 & Condition 4 & \\
\hline & & & & $\begin{array}{l}\text { MTF/MTF } \\
\text { decline ratio }\end{array}$ & $\begin{array}{l}\text { MTF/MTF } \\
\text { decline ratio }\end{array}$ & \\
\hline \multirow[t]{7}{*}{340} & 96 & 0.100 & 0.119 & $0.112 / 5.8 \%$ & $0.101 / 15.1 \%$ & $0.096 / 9.4 \%$ \\
\hline & 64 & 0.108 & 0.117 & $0.107 / 8.4 \%$ & $0.099 / 15.3 \%$ & $0.106 / 11.6 \%$ \\
\hline & 48 & 0.100 & 0.111 & $0.096 / 13.5 \%$ & $0.087 / 21.6 \%$ & $0.103 / 14.8 \%$ \\
\hline & 32 & 0.101 & 0.089 & $0.086 / 3.3 \%$ & $0.077 / 13.4 \%$ & $0.118 / 8.5 \%$ \\
\hline & 16 & 0.100 & 0.096 & $0.091 / 5.2 \%$ & $0.085 / 11.4 \%$ & $0.113 / 12.4 \%$ \\
\hline & 8 & 0.110 & 0.104 & $0.099 / 4.8 \%$ & $0.093 / 10.5 \%$ & $0.124 / 11.4 \%$ \\
\hline & Avg. & 0.1018 & 0.1060 & $0.098 / 6.8 \%$ & $0.090 / 14.6 \%$ & $0.11 / 11.3 \%$ \\
\hline \multirow[t]{7}{*}{265} & 96 & 0.104 & 0.100 & $0.095 / 5.0 \%$ & $0.076 / 24.0 \%$ & $0.093 / 9.7 \%$ \\
\hline & 64 & 0.100 & 0.103 & $0.095 / 7.7 \%$ & $0.076 / 26.2 \%$ & $0.104 / 10.3 \%$ \\
\hline & 48 & 0.092 & 0.097 & $0.086 / 11.3 \%$ & $0.076 / 21.6 \%$ & $0.097 / 17.1 \%$ \\
\hline & 32 & 0.097 & 0.090 & $0.086 / 4.4 \%$ & $0.078 / 13.3 \%$ & $0.099 / 13.9 \%$ \\
\hline & 16 & 0.100 & 0.096 & $0.091 / 5.2 \%$ & $0.087 / 9.3 \%$ & $0.105 / 12.5 \%$ \\
\hline & 8 & 0.120 & 0.113 & $0.111 / 1.7 \%$ & $0.105 / 7.7 \%$ & $0.109 / 12.1 \%$ \\
\hline & Avg. & 0.1021 & 0.0998 & $0.094 / 5.9 \%$ & $0.083 / 16.9 \%$ & $0.101 / 12.6 \%$ \\
\hline
\end{tabular}

Imaging condition 1 4 is designed for the qualification model of HiRIC while imaging condition 5 is designed for its flight model. Condition 1 represents MTFs of images acquired at the theoretical imaging frequency at the corresponding orbital altitude; Condition 2 represents MTFs of images acquired at the imaging frequency calculated from the real-time orbital parameters at the corresponding orbital altitude without errors; Conditions 3 5 represent the MTF of images acquired at the imaging frequency calculated from the real-time orbital parameters at the corresponding orbital altitude with orbital altitude errors of $1 \mathrm{~km}, 3 \mathrm{~km}$ and $2 \mathrm{~km}$, respectively

\section{Image Quality Analysis}

\subsection{Image Motion Compensation Effect}

As mentioned previously, a total of 48 sequence images of spatial frequency grating under different imaging conditions were obtained in this experiment. By calculating the dynamic MTF of these images, the image motion compensation effect of HiRIC was quantitatively evaluated. The results are recorded in Table 4.

From the results in the column of "Condition 1" in Table 4, It can be seen that when the motion speed of the imaging target, i.e., the rotation speed of the rotating motor, matches exactly with the theoretical imaging frequency of HiRIC, the average dynamic MTF of images obtained at different simulated orbital altitudes and TDI integration levels can reach more than 0.1. Since the static MTF of HiRIC images at Nyquist frequency is greater than 0.18 , its theoretical dynamic MTF at can reach about 0.114 , i.e., about 0.6325 times of the static MTF (Wang 1998). Considering a rotational error of about $\pm 1 \%$ of the rotating motor used in the test, and the installation error between the target movement direction and the camera imaging direction, the dynamic MTF will be worse than the theoretical value. Therefore, the dynamic MTF of HiRIC images obtained in this experiment is greater than 0.1 , which is within expectation, real and effective. This value will be used as the basis for comparison for subsequent experiments. 
According to the results in the column of "Condition 2" in Table 4, the dynamic MTF of HiRIC images obtained at different simulated orbital altitudes and TDI integration levels can also reach an average of about 0.1 when the imaging frequency is calculated from the realtime orbital parameters without errors, which is consistent with the MTF value of images obtained at the theoretical imaging frequency. Therefore, the algorithm for the in-orbit image motion compensation of HiRIC is correct and valid.

However, the dynamic MTF of HiRIC images started to show different degrees of degradation after adding different magnitudes of orbital altitude errors to the orbital parameters because the imaging frequency no longer matched the motion speed of the imaging target. The results in the column of "Condition 3" and "Condition 4" in Table 4 show that, the greater the orbital altitude error, the greater the decline of dynamic MTF of HiRIC images obtained at the simulated orbital altitude of $265 \mathrm{~km}$. It can be seen that when orbital altitude accuracy reaches $3 \mathrm{~km}$, the dynamic MTF of HiRIC images at different simulated orbital altitudes and TDI integration levels degraded by more than $10 \%$, and the corresponding image motion in the focal plane exceeded 0.5 pixels.

Combining the experience of HiRISE and HRSC, HiRIC requires the forward and lateral image motion in the focal plane should not exceed $1 / 2$ pixel respectively after compensation, and the corresponding dynamic MTF decline should not exceed $10 \%$. In order to ensure HiRIC meet the requirement of high resolution imaging, GRAS and CIOMP conducted another verification test on the image motion compensation effect using HiRIC flight model, which is listed in the column of "Condition 5" in Table 4. The test results show that when the orbital altitude error reaches $2 \mathrm{~km}$, the dynamic MTF of HiRIC images obtained at different simulated orbital altitudes and TDI integration levels will also decline by an average of over $10 \%$.

The image motion compensation effect evaluation test for HiRIC verifies the correctness and effectiveness of its in-orbit image motion compensation algorithm. The influence of different orbital altitude errors on the dynamic MTF of HiRIC images is evaluated. Compared with the image motion compensation effect of HiRISE and HRSC, HiRIC can obtain high quality images when the determination accuracy of orbital altitude is better than $2 \mathrm{~km}$. During the Tianwen-1 mission, the orbital altitude accuracy of the Orbiter can be reach about $1.5 \mathrm{~km}$, so the image motion compensation function of HiRIC can ensure that it obtains high-quality images.

\subsection{Focusing Effect}

A total of 5 sequence images have been obtained to evaluate the focusing effect of HiRIC by analyzing the static MTF of the images, as shown in Table 5.

In order to ensure the reliability and stability of the in-orbit imaging quality, the focal plane of HiRIC was positioned for an optimal imaging distance of $500 \mathrm{~km}$. This focal plane position can adapt to the imaging requirements when the orbital altitude is changed between 265 and $800 \mathrm{~km}$. According to the values in Table 5, the static MTF of images obtained at a simulated distance of $500 \mathrm{~km}$ is $0.205 @ 571 \mathrm{p} / \mathrm{mm}$ (Nyquist frequency of HiRIC). According to the specification, HiRIC requires an average static MTF of $\geq 0.18 @ 57 \mathrm{lp} / \mathrm{mm}$ in the CCD imaging area. Therefore, the static MTF of HiRIC meets the design requirements.

When the target is defocusing, the static MTF of HiRIC images for the infinity distance and $265 \mathrm{~km}$ simulation targets are 0.199@ 571p/mm and 0.201@ 57lp/mm, respectively, degraded by $2.92 \%$ and $1.95 \%$ according to the value of focus images. After focusing operation, the static MTF of HiRIC images for the same targets can be restored to the level of the images obtained at the optimal imaging distance of $500 \mathrm{~km}$, which is $0.205 @ 57 \mathrm{lp} / \mathrm{mm}$. 
Table 5 Static MTF of HiRIC images in the focusing effect test

\begin{tabular}{lllll}
\hline $\begin{array}{l}\text { Optimal } \\
\text { imaging } \\
\text { altitude }\end{array}$ & $\begin{array}{l}\text { Simulation } \\
\text { distance of } \\
\text { static target }\end{array}$ & $\begin{array}{l}\text { Static MTF } \\
\text { (@ Nyquist) }\end{array}$ & Deviation & $\begin{array}{l}\text { Static MTF } \\
\text { after focusing } \\
\text { (@ Nyquist) }\end{array}$ \\
\hline $500 \mathrm{~km}$ & Infinity & 0.199 & $2.92 \%$ & 0.205 \\
& $500 \mathrm{~km}$ & 0.205 & 0 & $/$ \\
& $265 \mathrm{~km}$ & 0.201 & $1.95 \%$ & 0.205 \\
\hline
\end{tabular}

The results in this paper show that the focusing operation of HiRIC is effective. In May 2021, Tianwen-1 Orbiter has entered the relay and survey orbit, and HiRIC has acquired high-resolution images of the landing area, which shows that the focal plane position set on the ground can ensure the need to acquire high-quality images. Subsequently, we will choose whether to apply the focusing function or not according to the actual working condition of HiRIC in orbit, otherwise we plan to keep the current focal plane position unchanged.

\subsection{Image Quality of the External Field Imaging Experiment}

The feasibility and effectiveness of the in-orbit detection process of HiRIC is evaluated by the external field imaging experiment. Images of this experiment can be used as reference for analyzing the quality of in-orbit images. Here we evaluate the image quality of the external field imaging experiment through compression quality, radiation correction effect, and CCD splicing effect.

\subsubsection{Data Compression Quality}

Due to the huge data volume and the limited data transmission capability of Mars exploration mission, HiRIC images must be transmitted after compression.

HiRIC images are compressed using the JPEG2000 algorithm, which is currently recognized as the best compression algorithm in the field of still image compression. For remote sensing images, the recovered images obtained by JPEG2000 compression have richer feature details and textures, and better Peak Signal-to-Noise Ratio (PSNR) than other still image compression algorithms, such as SPIHT (Taubman and Marcellin 2001; Rabbani and Joshi 2002). The JPEG2000 algorithm first performs a discrete wavelet transform on the raw images, and then quantizes and entropy codes the transform coefficients (EBCOT) before forming the output bit stream. After the compressed image data is stored or transmitted, entropy decoding, inverse quantization, and inverse wavelet transform are performed to recover the image data.

Lossy compression will cause loss of image texture details and affect HiRIC image quality. An optimal image data compression ratio under the premise of ensuring the data transmission ability is very important for the image quality of HiRIC.

The main index of image data compression quality evaluation is PSNR, which is measured in decibels $(\mathrm{dB})$. During the in-orbit operation, HiRIC performs data transmission with the Orbiter platform every 512 image lines. Under the compression mode, the images will be compressed in blocks at a minimum resolution of 384 pixels/line so that there will be a total of 16 types of compressed blocks, which recorded as "resolution 1 16" with the pixel number of $(384 * \mathrm{~N}) * 512(\mathrm{~N}=1,2,3 \ldots 16)$. In order to evaluate the data compression quality, 18 scene HiRIC panchromatic images in the external field imaging experiment which processed by compression and decompression were selected to analyze the PSNR (seen in Table 6). 
Table 6 Compression test results

\begin{tabular}{|c|c|c|c|c|c|c|c|c|}
\hline \multirow{2}{*}{$\begin{array}{l}\text { Data } \\
\text { type }\end{array}$} & \multirow{2}{*}{\multicolumn{2}{|c|}{$\begin{array}{l}\text { Data } \\
\text { volume }\end{array}$}} & \multicolumn{6}{|l|}{ PSNR } \\
\hline & & & $\begin{array}{l}\text { lossless } \\
\text { compression }\end{array}$ & $\begin{array}{l}3: 1 \\
\text { compression }\end{array}$ & $\begin{array}{l}4: 1 \\
\text { compression }\end{array}$ & $\begin{array}{l}6: 1 \\
\text { compression }\end{array}$ & $\begin{array}{l}12: 1 \\
\text { compression }\end{array}$ & $\begin{array}{l}15: 1 \\
\text { compression }\end{array}$ \\
\hline \multicolumn{2}{|c|}{$\begin{array}{l}\text { "resolution 1" } \\
(384 * 512)\end{array}$} & 96 & INF & 70.97 & 70.52 & 64.49 & 60.09 & 59.42 \\
\hline \multicolumn{2}{|c|}{$\begin{array}{l}\text { "resolution } 2 " \\
(768 * 512)\end{array}$} & 96 & INF & 71.83 & 71.78 & 66.67 & 63.09 & 62.45 \\
\hline \multicolumn{2}{|c|}{$\begin{array}{l}\text { "resolution 4" } \\
(1536 * 512)\end{array}$} & 144 & INF & 70.79 & 69.16 & 64.17 & 60.08 & 59.38 \\
\hline \multicolumn{2}{|c|}{$\begin{array}{l}\text { "resolution 8" } \\
(3072 * 512)\end{array}$} & 72 & INF & 71.55 & 71.35 & 65.90 & 62.05 & 61.38 \\
\hline \multicolumn{2}{|c|}{$\begin{array}{l}\text { "resolution 16" } \\
(6144 * 512)\end{array}$} & 90 & INF & 71.80 & 71.57 & 66.28 & 62.16 & 61.47 \\
\hline
\end{tabular}

Typically, the values of PSNR, for lossy compression of an 8 bits bitdepth image, are between 30 and $50 \mathrm{~dB}$ and when the PSNR is greater than $40 \mathrm{~dB}$, then the two images are indistinguishable (Welstead 1999; Manel et al. 2016). HiRIC offers 12-bits bitdepth, which is different from the classical 8-bits bitdepth images. It can be seen from the results that almost all the PSNRs of the test images under different compression ratios are always greater than $60 \mathrm{~dB}$, even if compression ratio is greater than $6: 1$. One possible reason is that the digital numbers (DNs) of images obtained in the external field imaging test is low relative to those of the 12-bits bitdepth images, resulting in little variation between the pre- and postcompressed images which makes the PSNR too large. Due to the lack of sufficient research on the optimal PSNR of 12 bit quantized images, it is clear that PSNR is no longer an optimal measure of the compression quality of these images. Therefore, there are insufficient evidence to determine the optimal compression ratio of HiRIC images based on the PSNRs results.

PSNR is an objective indicator to evaluate the effect of image compression, but it is based on the error between corresponding pixels, that is, based on error-sensitive image quality evaluation. Because it is not fully applicable to 12-bit quantized images, PSNR maybe not be the best evaluation indicator for this experiment. In order to further analyze the reasonable compression ratio for HiRIC images during the in-orbit operation, an index named structural similarity (SSIM, Di and Liu 2006) is introduced here to quantitatively analyze the compression quality of images, together with PSNR.

SSIM is a measure of how similar two images are. It was first proposed by the Laboratory for Image and Video Engineering at the University of Texas at Austin, using two images, one uncompressed and undistorted and the other distorted. Like PSNR, SSIM is often used as an evaluation of image quality (Zhou et al. 2004). SSIM can quantify image quality degradation caused by processing such as data compression or by losses in data transmission. The value range is $0 \sim 1$, and when the two images are completely consistent, the SSIM value is equal to 1

Figure 4 reflect the effect of data compression on the similarity of HiRIC images. The SSIM values under every compression ratio are less than 1 . On the other hand, when the compression ratio is greater than 6:1, the SSIM values show a rapid decline, and the similarity between images becomes worse. Combined with the PSNR analysis results, when the compression ratio is $6: 1$, the PSNR of the test images are greater than 60 . Therefore, it is 


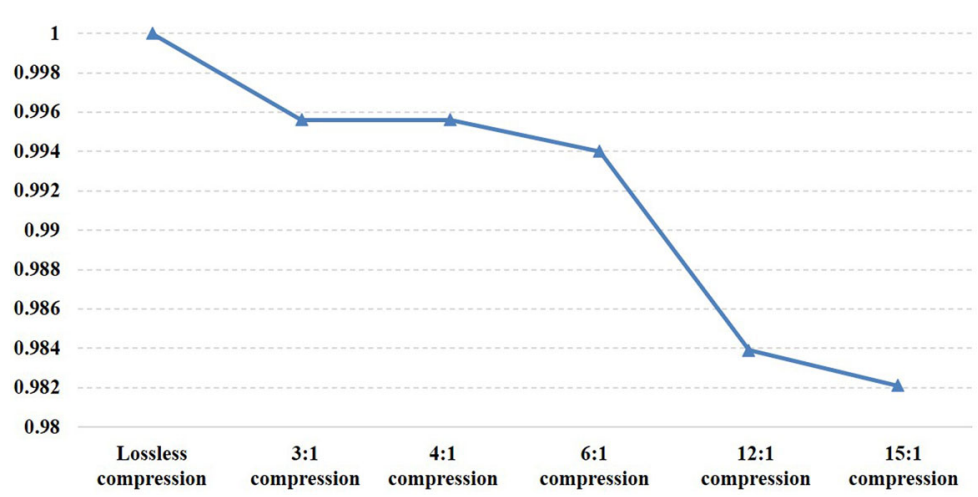

Fig. 4 SSIM values of HiRIC images at different compression ratios

recommended that the compression ratio does not exceed 6:1 during the in-orbit operation to ensure the image quality.

\subsubsection{Radiation Correction Effect}

Radiation correction is the main content of HiRIC image data pre-processing to eliminate the inconsistency of each pixel's response due to CCDs defects, and to avoid the pixel response non-uniformity (PRNU) in uniform illumination conditions.

The radiation correction is performed by a set of matrix coefficients for the DN value of each TDI CCD pixel, including the slope $k$ and the intercept $a$. These coefficients are given by the development department through radiation calibration in the laboratory, taking fully into account the radiation correction bias between the CCDs and the temperature dependence in dark conditions (Meng et al. 2021). The radiometric correction coefficients will be applied to the production of HiRIC scientific data products of Tianwen-1 mission in the PDS4 version format (Tan et al. 2021). This paper will focus on whether the application of the radiation correction coefficients meets the needs of scientific applications.

The HiRIC has 6144 pixels per CCD panchromatic image, divided into 4 output channels, 1536 pixels per channel (Meng et al. 2021). In order to evaluate the radiation calibration effect of HiRIC, a comparison of the images obtained in the external field imaging test before and after radiation correction is shown in Fig. 5. It can be seen that through the relative radiation correction, the consistency of the $\mathrm{DN}$ for different output channels has been improved, but there are still some grayscale deviations. The main reason is that the radiation calibration algorithm for the HiRIC qualification mode is not well adapted to the differences between CCDs output channels, and the correction of different luminance conditions is not perfect. On the other hand, the DN of the images obtained in the external field push-scan test is low as mentioned above, which will also cause the radiation calibration coefficient not suitable for the non-uniformity between images.

In response to the problems reflected in the external field imaging test, the algorithm of radiation calibration for HiRIC flight model was optimized. The values of the radiation correction coefficients $k$ and $a$ are calculated by the least squares fitting, while the calibration process is uniformly corrected for all out put channels of the three CCDs. Therefore, the radiation correction effect of images for the flight model can be significantly improved, and the correction uniformity can be increased by about $50 \%$ compared with the qualification mode. The relative radiation correction uncertainty of panchromatic images of HiRIC flight model is better than $3 \%$ (seen in Fig. 6). 


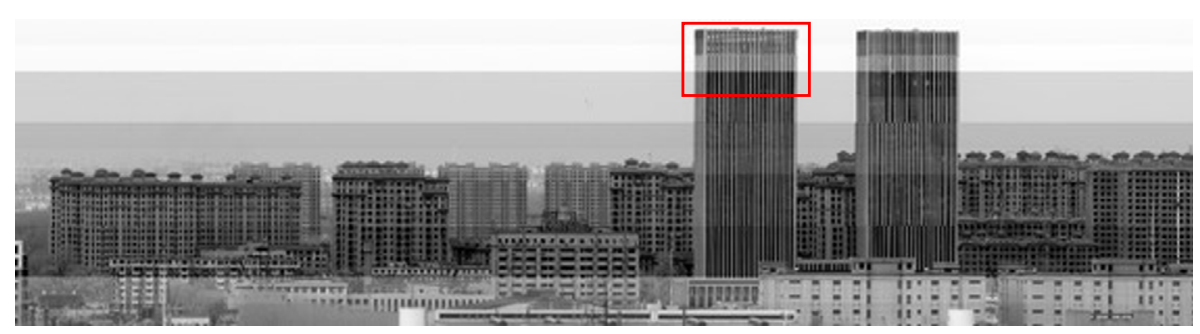

(a)

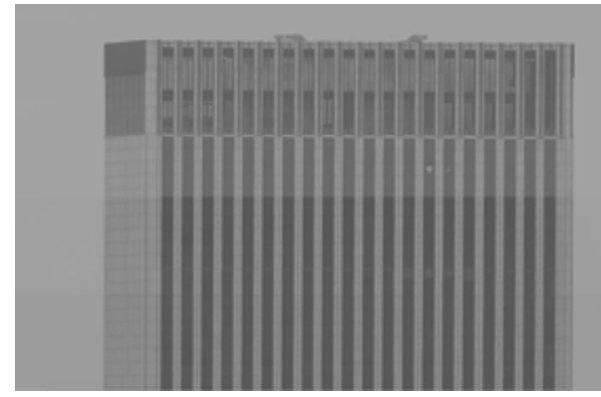

(b)

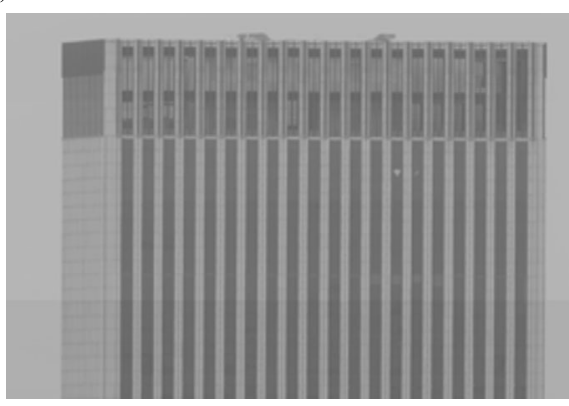

(c)

Fig. 5 Radiation correction effect of HiRIC qualification model images. (a) is a mosaic of the 3 CCD images obtained in the external field push-scan test. (b) and (c) are images before and after radiation correction corresponding to the scene in the red box in (a). It can be seen that there are still some grayscale deviations between output channels

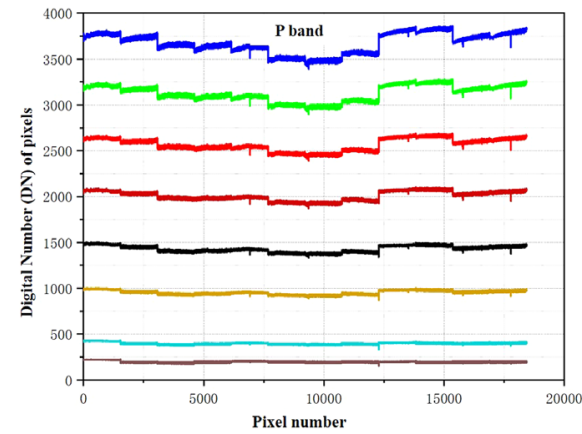

(a)

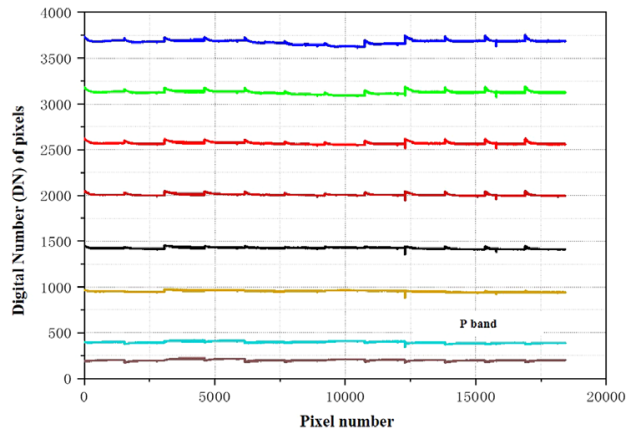

(b)

Fig. 6 Radiation correction effect of HiRIC flight model. (a) and (b) are the comparison of DNs for panchromatic band images under different luminance conditions before and after radiation correction. The relative radiation correction uncertainty is better than $3 \%$

\subsubsection{CCD Stitching Accuracy}

In order to obtain a larger field of view and effective swath width, high resolution cameras are usually assembled using multiple staggered TDI CCDs in the same focus plane. For example, HiRISE has 14 CCDs and the staggered CCDs overlapped by $48 \pm 5$ pixels with adjacent CCDs in the same color band pass to provide an effective swath width of $\sim 20,048$ 


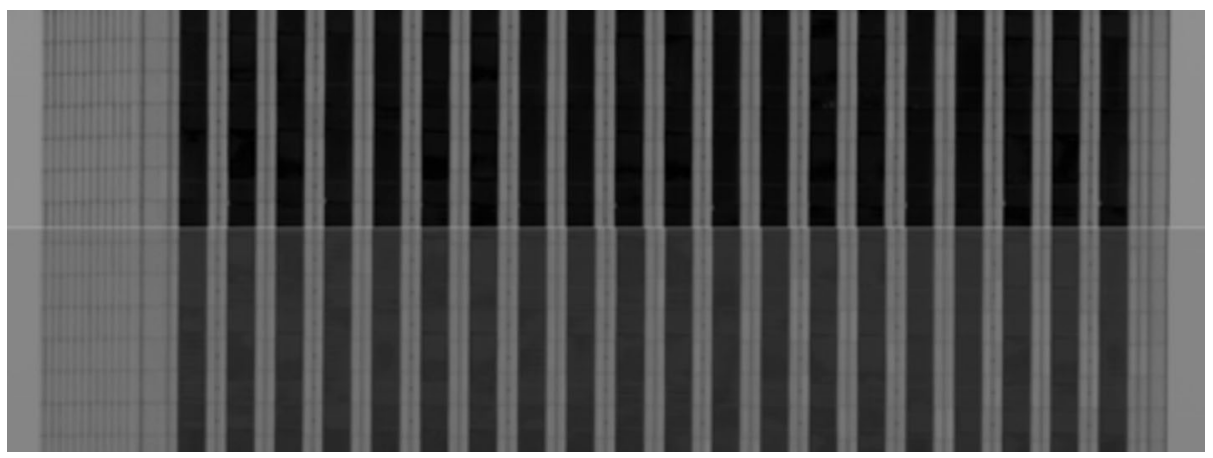

Fig. 7 Stitching results of HiRIC qualification model image. In the middle of the image, the overlapping part of the two CCDs, the scene appears obvious misaligned

pixels for the RED images, and 4,048 pixels for the blue-green and NIR images (McEwen et al. 2010).

HiRIC is also composed of three staggered TDI CCDs. Adjacent CCDs are assembled with an overlap of 116 pixels (panchromatic band). Because the images obtained during each integration period is not a continuous and complete scan line, but is divided into three separate records, the stitching accuracy between the three CCDs directly affects the quality of the final HiRIC images. Again, geometric calibration parameters such as the coplanarity of the staggered TDI CCDs, their parallelism and overlap dimensions have been rigorously calibrated in the laboratory by the development department (Meng et al. 2021). In this paper, the stitching of panchromatic images obtained in the external field imaging test was performed and the stitching accuracy was analyzed using the calibration results of the CCD stitching parameters of the HiRIC qualification model.

It can be seen from Fig. 7 that the stitched images are misaligned. The main reason is that the splicing of CCDs for HiRIC qualification model has not reached the optimal state. The flatness of the focal plane has a maximum deviation of about 1.9 pixels, and the collinearity of the focal plane has a maximum deviation of about 2.7 pixels which will cause the deviation of stitched images. On the other hand, factors such as camera's attitude, the deviation of the drift angle control, and the change in the distance from the imaging target during the external field imaging test further exacerbate the deviation between stitched images.

In response to the problems reflected above, the CCD linear array stitching parameters were accurately measured during the calibration of HiRIC flight model. The calibration results are shown in Table 7.

It can be seen that the mechanical stitching accuracy of staggered CCDs on the focal plane of the HiRIC flight model is better than $0.00125 \mathrm{~mm}$, which is corresponding to 0.15 pixels. The calibration accuracy (standard deviation) of the CCD mechanical stitching state is better than $1 \mu \mathrm{m}$, that is, 0.11 pixels, which meet the requirement of high resolution imaging quality of HiRIC.

The geometric calibration results in Table 7 are the actual measurements of the focal plane design installation layout in Fig. 2, which verifies the actual installation of HiRIC. In the subsequent scientific application process, in order to map HiRIC images to the cartographic systems for Mars, it is necessary to combine various information such as camera focal length, image principal point, HiRIC mounting parameters on the Orbiter, and the or- 
Table 7 Calibration results of CCD splicing parameters for HiRIC flight model

\begin{tabular}{ll}
\hline Contents & Calibration results \\
\hline Splicing coplanarity & Better than $\pm 0.00125 \mathrm{~mm}$ \\
Splicing parallelism & $\begin{array}{l}\text { Observe along }-\mathrm{Z} \text { axis direction, CCD_1 relative to CCD_2 around Z axis } \\
\text { counterclockwise rotation 5.7" } \\
\text { Observe along }-Z \text { axis direction, CCD_3 relative to CCD_2 around Z axis } \\
\text { counterclockwise rotation 3.8 }\end{array}$ \\
& The maximum value is $0.0005 \mathrm{~mm}$ \\
Splicing collinearity & CCD_1 and CCD_21.0130 $\mathrm{mm}(119$ pixels $)$ \\
Overlap size & CCD_3 and CCD_21.0105 mm (119 pixels) \\
\hline
\end{tabular}

bit and attitude of the Orbiter, in addition to stitching parameters, to establish a rigorous imaging geometry model of HiRIC.

\section{Discussion}

\subsection{Estimated Detection Capability of HiRIC During in-Orbit Operation}

In this paper, special experiments were designed to fully validate the in-orbit detection capability and performance of HiRIC. The main conclusions are as follows.

- Through image motion compensation, the dynamic MTF of HiRIC images will not degrade by more than $10 \%$ when the orbital altitude accuracy of Tianwen-1 Orbiter is better than $2 \mathrm{~km}$, and the corresponding forward (along the flight direction) and lateral (perpendicular to the flight direction) image motions of the imaging target in the focal plane will not exceed 0.5 pixels.

- The focusing function of HiRIC meets requirements of high resolution imaging on the large elliptical orbit of Tianwen-1. In the planned imaging orbit altitude range of $265 \mathrm{~km} \sim 800 \mathrm{~km}$, the static MTF of HiRIC images will not degrade by more than $3 \%$ due to defocusing.

- The in-orbit detection process for HiRIC is effective and valid images can be obtained in external field imaging tests.

- The PSNR of images in external field imaging tests processed by compression and decompression is greater than $60 \mathrm{db}$, even if the compression ratio is larger than 6:1. Considering that HiRIC images are 12-bit quantized, combined with the results of the analysis of the image structural similarity of images (SSIM), the compression ratio of HiRIC is not recommended to exceed 6:1 during in-orbit operation.

- The radiation calibration method of HiRIC flight model has been adjusted through ground scientific verification test, and the uniformity of the radiation correction can be improved by about $50 \%$ over its qualification model.

- Similarly, the geometric calibration method of HiRIC flight model has also been adjusted and the stitching accuracy can be better than 0.5 pixels (panchromatic band).

It can be seen that, the technical specifications of HiRIC meet the design requirements, and the performance status is stable, with the ability to obtain high resolution images of the Martian surface. It should be noted that, due to the limitations of ground test conditions, not all the factors that affect the imaging quality of HiRIC can be verified through experiments, 
Table 8 Calibration results of CCD splicing parameters for HiRIC flight model

\begin{tabular}{|c|c|c|c|c|}
\hline \multirow{3}{*}{$\begin{array}{l}\text { Orbital } \\
\text { altitudes }(\mathrm{km})\end{array}$} & \multicolumn{4}{|l|}{ The ground albedo } \\
\hline & 0.05 & 0.10 & 0.2 & 0.30 \\
\hline & $\begin{array}{l}\text { Integration levels } \\
\text { /SNR }\end{array}$ & $\begin{array}{l}\text { Integration levels } \\
\text { /SNR }\end{array}$ & $\begin{array}{l}\text { Integration levels } \\
\text { /SNR }\end{array}$ & $\begin{array}{l}\text { Integration levels } \\
\text { /SNR }\end{array}$ \\
\hline 265 & $96 / 92.4$ & $96 / 100.5$ & $96 / 115.4$ & $64 / 100.3$ \\
\hline 315 & $96 / 108.2$ & $96 / 117.3$ & $64 / 104.3$ & $64 / 117.1$ \\
\hline 365 & $96 / 120.6$ & $64 / 101.3$ & $64 / 116.4$ & 48/109.1 \\
\hline 415 & $64 / 102.9$ & $64 / 111.7$ & $48 / 107.0$ & $48 / 120.0$ \\
\hline 465 & $64 / 112.6$ & $48 / 101.8$ & 48/101.6 & $32 / 101.6$ \\
\hline 515 & $48 / 101.7$ & $48 / 110.3$ & $48 / 126.4$ & $32 / 110.2$ \\
\hline
\end{tabular}

such as the ground albedo of the Martian surface and the variation of the solar elevation angle which will affect the SNR of HiRIC images. For these factors, we performed a theoretical analysis of the influence situation.

For specific areas on the Martian surface, the higher the ground albedo and the greater the solar elevation angle, the higher the SNR of HiRIC images. According to the results of OMEGA on the Mars Express and Compact Reconnaissance Imaging Spectrometer (CRISM) on the MRO, the bolometric albedo variation on the Martian surface ranges from 0.08 to 0.35 (Vincendon et al. 2015). Based on this background, simulations were performed to calculate the SNR and corresponding optimal TDI integration levels of HiRIC images at different orbital altitudes, ground albedos, and solar elevation angles. Results show that for the case where the ground albedo varies from 0.05 to 0.30 , the imaging condition of HiRIC image SNR greater than 100 can be satisfied by setting different TDI integration levels (32 96) when the solar elevation angle is greater than $15^{\circ}$ (seen in Table 8). According to the orbit design of Tianwen-1 mission, HiRIC will carry out detection near the periareon, i.e., in the orbital altitude range of about $265 \mathrm{~km} \sim 800 \mathrm{~km}$, and the solar altitude angle will always remain greater than $15^{\circ}$ during this orbital segment. Therefore, ground albedo and solar elevation angle can meet the requirement of image quality and detection capability of HiRIC after determining the imaging targets and imaging epoch.

\subsection{Proposals for in-Orbit Imaging Strategy}

The results of these experiments showed that the performance status of HiRIC meets the requirements of obtaining high resolution images on the Martian surface. However, the actual in-orbit imaging process of HiRIC will be more complicated and challenged. HiRIC will not start its work until Mars parking orbit phase of Tianwen-1 mission (Wu et al. 2013), when it will obtain stereo images of the candidate landing site for terrain reconstruction to support the safe landing of the "ZhuRong" rover and its scientific detection on the Martian surface. Then, HiRIC will image areas of interest on the Martian surface together with the 8 Martian landing sites of international Mars exploration missions in the scientific exploration phase (Kirk et al. 2008; Golombek et al. 2012; Fergason et al. 2017).

HiRIC has only one effective imaging linear array (staggered by three TDI CCDs) with a swath width of only $9 \mathrm{~km} @ 265 \mathrm{~km}$ so that the Orbiter needs to adjusted its attitude in yaw and pitch direction to realize stereo coverage on the Martian surface by multiple imaging. Therefore, it is also crucial to formulate the in-orbit imaging strategy based on the actual situation of the mission. 
Fig. 8 Coverage of HiRIC images on landing site. The alternating yellow and green boxes in indicate 9 imaging strips, with an overlap coverage of $15 \%$, and the red box indicates the $150 \mathrm{~km}$ (north-south direction $) \times 60 \mathrm{~km}$ (east-west direction) landing area

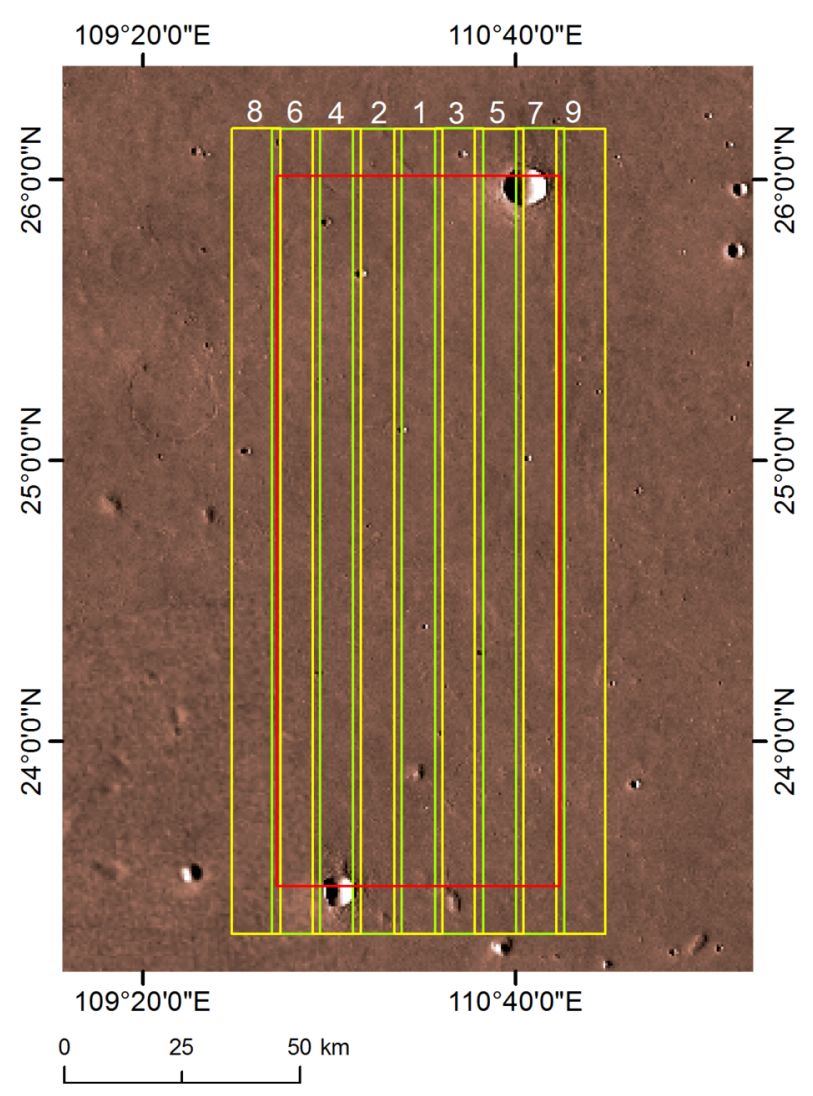

The landing area of Tianwen-1 mission is $150 \mathrm{~km}$ (north-south, or along the latitude direction) $\times 60 \mathrm{~km}$ (east-west, or along the longitude direction), as shown in Fig. 8. As previously stated, HiRIC uses panchromatic band CCD push-scan imaging as the default detection mode, and the imaging swath width is $9 \mathrm{~km} @ 265 \mathrm{~km}$. In order to complete the stereo imaging of the landing area, the HiRIC needs to image the same area of the landing area along the north-south direction by means of multi-tracks stereo, with 2 angles from the forward- and backward- view, and the imaging baseline-height ratio $\geq 0.4$, i. e., the forward- and backward- view pitch angle of the Orbiter should be $11.31^{\circ}$; in addition, the image overlap coverage along the east-west direction should be no less than $15 \%$, therefore, 9 imaging strips were designed according to the HiRIC swath width to achieve full coverage of the landing area with stereo images. As a result, a total of at least 18 images are needed for our mission (forward- and backward- view images are needed for the same area in Fig. 8 to obtain stereo images). The adjustment ranges of yaw and pitch angle for the Orbiter to achieve stereo coverage will be no larger than $\pm 20^{\circ}$.

For the imaging task of areas of interest on the Martian surface and the currently 8 Martian landing sites of international Mars exploration missions, the Orbiter attitude are not needed to adjusted any more. HiRIC will image these targets when the Orbiter performs an overflight. The analysis results show that solar elevation angle when HiRIC works will be changed from $15^{\circ}$ to $70^{\circ}$ and TDI integration levels from 32 to 96 can be used to obtain high resolution images, in which TDI integration levels of 64 will be most commonly used. 
HiRIC is another high resolution optical camera for Mars exploration. In this paper, special experiments are designed to verify the actual detection capability and performance of HiRIC in the simulation exploration environment based on the in-orbit detection conditions of Tianwen-1 mission. The test results are effective complements to the HiRIC laboratory calibration tests. On the other hand, recommendations are made for its in-orbit imaging strategies to ensure the acquisition of high-quality images. In the future, HiRIC is expected to obtain detailed images of areas of interest on the Martian surface with higher resolution and larger coverage, which can serve as an effective complementation to the existing Martian high resolution images, enrich the global coverage of Mars sub-meter-scale high-resolution images, and further deepen our comprehensive understanding of the Mars.

Acknowledgements This research was funded by Beijing Municipal Science and Technology Commission (NO. Z191100004319001), and the Specialized Research Fund for Shandong Provincial Key Laboratory. We thank the team of the Tianwen-1 Project for their successful work, especially the GRAS of CLEP and CIOMP for their valuable and efficient assistance with providing the data and data calibration.

Open Access This article is licensed under a Creative Commons Attribution 4.0 International License, which permits use, sharing, adaptation, distribution and reproduction in any medium or format, as long as you give appropriate credit to the original author(s) and the source, provide a link to the Creative Commons licence, and indicate if changes were made. The images or other third party material in this article are included in the article's Creative Commons licence, unless indicated otherwise in a credit line to the material. If material is not included in the article's Creative Commons licence and your intended use is not permitted by statutory regulation or exceeds the permitted use, you will need to obtain permission directly from the copyright holder. To view a copy of this licence, visit http://creativecommons.org/licenses/by/4.0/.

\section{References}

N. Bondarenko, I. Dulova, Y. Kornienko, Topography of polygonal structures at the Phoenix landing site on Mars through the relief retrieval from the HiRISE images with the improved photoclinometry method. Sol. Syst. Res. 48(4), 243-258 (2014)

M.H. Carr, The Surface of Mars (Cambridge University Press, New York, 2007)

W.A. Delamere et al., Colour imaging of Mars by the High Resolution Imaging Science Experiment (HiRISE). Icarus 205, 38-52 (2010). https://doi.org/10.1016/j.icarus.2009.03.012

H.W. Di, X.F. Liu, Image fusion quality assessment based on structural similarity. Acta Photonica Sin. 35(5), 766-771 (2006) (in Chinese)

C. Dundas, A. McEwen, S. Diniega et al., New and recent gully activity on Mars as seen by HiRISE. Geophys. Res. Lett. 37(7), L07202 (2010)

R. Fergason, R. Kirk, G. Cushing et al., Analysis of local slopes at the InSight landing site on Mars. Space Sci. Rev. 211(1-4), 109-133 (2017)

M. Golombek, J. Grant, D. Kipp et al., Selection of the Mars Science Laboratory landing site. Space Sci. Rev. 170(1-4), 641-737 (2012)

K. Gwinner, R. Jaumann, E. Hauber et al., The High Resolution Stereo Camera (HRSC) of Mars Express and its approach to science analysis and mapping for Mars and its satellites. Planet. Space Sci. 126, 93-138 (2016)

R. Jaumann, The High Resolution Stereo Camera (HRSC) experiment on Mars Express: instrument aspects and experiment conduct from interplanetary cruise through the nominal mission. Planet. Space Sci. 55(7-8), 928-952 (2007)

L. Keszthelyi, W. Jaeger, A. McEwen et al., High Resolution Imaging Science Experiment (HiRISE) images of volcanic terrains from the first 6 months of the Mars Reconnaissance Orbiter primary science phase. J. Geophys. Res., Planets 113(E4), E04005 (2008)

R. Kirk, E. Howington-Kraus, M. Rosiek et al., Ultrahigh resolution topographic mapping of Mars with MRO HiRISE stereo images: meter-scale slopes of candidate Phoenix landing sites. J. Geophys. Res., Planets 113(E3), E00A24 (2008)

S.W. Lee, E.D. Skulsky, J.D. Chapel et al., Mars Reconnaissance Orbiter design approach for high resolution surface imaging, in 26th Annual AAS Guidance and Control Conference (Am. Astron. Soc., Breckenridge, 2001) 
A. Lefort, P. Russell, N. Thomas et al., Observations of periglacial landforms in Utopia Planitia with the High Resolution Imaging Science Experiment (HiRISE). J. Geophys. Res., Planets 114(E4), E04005 (2009)

C. Li, R. Zhang, D. Yu et al., China's Mars exploration mission and science investigation. Space Sci. Rev. 217, 57 (2021)

J.J. Liu, Y. Su, W. Zuo et al., Ground research and application system of China's first Mars exploration mission. J. Deep Space Explor. 5(5), 414-425 (2018)

M.C. Malin, G.E. Danielson, A.P. Ingersoll et al., Mars observer camera. J. Geophys. Res. 97(E5), 7699-7718 (1992)

M.C. Malin, J.F. Bell, B.A. Cantor et al., Context camera investigation on board the Mars Reconnaissance Orbiter. J. Geophys. Res. 112, E05S04 (2007). https://doi.org/10.1029/2006JE002808

M.C. Malin, K.S. Edgett, B.A. Cantor et al., An overview of the 1985-2006 Mars Orbiter Camera science investigation. Mars 5, 1-60 (2010)

D. Manel, B. Belgacem et al., An enhencment medical image compression algorithm based on neural network. Int. J. Adv. Comput. Sci. Appl. 7(5), 484-489 (2016)

T.B. McCord, J.B. Adams, G. Bellucci et al., Mars express high resolution stereo camera spectrophotometric data: characteristics and science analysis. J. Geophys. Res., Planets 112, E06004 (2007)

A.S. McEwen, E.M. Eliason, J.W. Bergstrom et al., Mars Reconnaissance Orbiter's High Resolution Imaging Science Experiment (HiRISE). J. Geophys. Res. 112, E05S02 (2007). https://doi.org/10.1029/ 2005JE002605

A. McEwen, M. Banks, N. Baugh et al., The High Resolution Imaging Science Experiment (HiRISE) during MRO's Primary Science Phase (PSP). Icarus 205, 2-37 (2010)

Q. Meng, D. Wang, X.D. Wang et al., High Resolution Imaging Camera (HiRIC) on China's First Mars Exploration Tianwen-1 mission. Space Sci. Rev. 217, 42 (2021)

NASA Technical Reports Server, Mariner Mars 1971 project final report (2011)

NASA, Program \& Missions Historical Log (2019). https://mars.nasa.gov/mars-exploration/missions/ historical-log/

G. Neukum, R. Jaumann, The HRSC Co-Investigator Team, In HRSC: The High Resolution Stereo Camera of Mars Express, ESASP-1240 (2004), p. 19

J. Oberst, G. Schwarz, T. Behnke et al., The imaging performance of the SRC on Mars Express. Planet. Space Sci. 56, 473-491 (2008)

M. Rabbani, R. Joshi, An overview of the JPEG 2000 still image compression standard. Signal Process. Image Commun. 17, 3-48 (2002)

X. Ren, J.J. Liu, C.L. Li et al., A global adjustment method for photogrammetric processing of Chang'E-2 stereo images. IEEE Trans. Geosci. Remote Sens. 57(9), 6832-6843 (2019)

X. Tan, J. Liu, X. Zhang et al., Design and validation of the scientific data products for China's Tianwen-1 mission. Space Sci. Rev. 217, 69 (2021)

D. Taubman, M. Marcellin, JPEG 2000: Image Compression Fundamentals, Standards, and Practice (Kluwer, Norwell, 2001)

N. Thomas, G. Cremonese, R. Ziethe et al., The colour and stereo surface imaging system (CaSSIS) for the ExoMars Trace Gas Orbiter. Space Sci. Rev. 212, 1897-1944 (2017)

M. Vincendon, J. Audouard, F. Altieri et al., Mars Express measurements of surface albedo changes over 2004-2010. Icarus 251, 145-163 (2015)

J.Q. Wang, Overall Design of Optical Instruments. Changchun Institute of Optics, Fine Mechanics and Physics, Chinese Academy of Sciences, Changchun (1998)

S.T. Welstead, Fractal and Wavelet Image Compression Techniques (SPIE, Bellingham, 1999), pp. 155-156. ISBN 978-0-8194-3503-3

W.R. Wu, Z.Y. Pei T.J. Liu et al., Chang'e 3 Engineering Technical Manual (China Astronautic Publishing House, Beijing, 2013)

P.J. Ye, Z.Z. Sun, W. Rao et al., Mission overview and key technologies of the first Mars probe of China. Sci. China, Technol. Sci. 60, 649-657 (2017)

W. Zhou, A.C. Bovik, H.R. Sheikh et al., Image quality assessment: from error visibility to structural similarity. IEEE Trans. Image Process. 13(4), 600-612 (2004)

Publisher's Note Springer Nature remains neutral with regard to jurisdictional claims in published maps and institutional affiliations. 